\title{
Effects of Duct Cross Section Camber and Thickness on the Performance of Ducted Propulsion Systems for Aeronautical Applications
}

\author{
Rodolfo Bontempo and Marcello Manna \\ Dipartimento di Ingegneria Industriale, Università degli Studi di Napoli Federico II, Via Claudio 21, 80125 Napoli, Italy \\ Correspondence should be addressed to Marcello Manna; marcello.manna@unina.it
}

Received 11 August 2015; Revised 18 January 2016; Accepted 19 January 2016

Academic Editor: Christopher J. Damaren

Copyright (C) 2016 R. Bontempo and M. Manna. This is an open access article distributed under the Creative Commons Attribution License, which permits unrestricted use, distribution, and reproduction in any medium, provided the original work is properly cited.

The axisymmetric flow field around a ducted rotor is thoroughly analysed by means of a nonlinear and semi-analytical model which is able to deal with some crucial aspects of shrouded systems like the interaction between the rotor and the duct, and the slipstream contraction and rotation. Not disregarding the more advanced CFD based methods, the proposed procedure is characterised by a very low computational cost that makes it very appealing as analysis tool in the preliminary steps of a design procedure of hierarchical type. The work focuses on the analysis of the effects of the camber and thickness of the duct cross section onto the performance of the device. It has been found that an augmentation of both camber and thickness of the duct leads to an increase of the propulsive ideal efficiency.

\section{Introduction}

Ducted fans and propellers have had some success in aeronautical applications especially by virtue of the higher thrust to power ratio offered by these propulsive systems compared to an open propeller with identical diameter. Consequently, all vehicles requiring a higher value of the propulsive efficiency at low speed are often equipped with ducted rotors. The most remarkable examples refer to V/STOL aircraft, compound helicopters, autogyros, airboats, hovercraft, seaplanes, and airships. Beyond the improvement in the propulsive efficiency, other advantages are related to the reduction of the tip losses (especially in hovering condition) and of the noise. However, this kind of machine has never known a wide success in the aeronautical applications partly because of the high cost and weight and partly because of the impossibility to achieve an optimal design for a wide range of advance speeds. For these reasons, in the earlier years, many experimental studies have attempted to enhance the understanding of the operating principles of shrouded rotors (see, e.g., [1-6]). More recently, Abrego and Bulaga [7] experimentally analysed the performance of a ducted fan in the 7-by-10-foot wind tunnel at the NASA Ames Research Center. The model consisted of a 5-bladed fixed pitch propeller (37.8 in diameter), a duct with a ratio between the chord and the rotor diameter roughly equal to 0.26 , and, finally, a 3 -in chord outlet exit vane with manually adjustable 1 -in chord flaps. The effects of different duct geometrical parameters were investigated for both hover and forward flight conditions. In the latter case, the angle $\alpha$ between the flight direction and the axis of the machine was varied in the range $\left[85^{\circ}, 115^{\circ}\right]$. The authors found that the propulsive force decreases with the angle $\alpha$. Moreover, the side forces experienced by the outlet guide vane were significantly affected by the flap deflection. These aspects are particularly important from the flight control point of view. In fact, if the ducted fan is sufficiently above or below the vehicle gravity centre, those side forces can be used to produce control moments. Finally, the extension of the duct chord was found to have marginal effects on the propulsive thrust. Oweis et al. $[8,9]$ carried out an extensive experimental campaign to investigate the most important physical aspects of the tip-leakage flow in ducted propellers. The data were acquired by means of both threedimensional laser Doppler and particle image velocimetry. They highlighted the presence of multiple vortices in the blade tip wake, where the largest vortex was associated with 
the tip-leakage. The data also show that the relative strength of the primary tip vortex of ducted propellers is reduced when compared to open propellers. Graf et al. [10] investigated by experimental means the effect of the duct lip shape on the performance of ducted fans. They found that a small leading edge curvature radius gives rise to a better performance in forward conditions, while a large leading edge radius has to be preferred in hovering conditions. Akturk et al. $[11,12]$ used a planar particle image velocimetry system to investigate the duct aerodynamic performance in hover and forward flight. In Koc et al. [13], the effect of the duct geometry on ducted propeller performance in axial flight was experimentally studied. In particular, velocity profiles and thrust and power coefficients obtained by using NACA 0012, 0018, and 4312 are compared with open propeller results. Yilmaz et al. [14] experimentally investigated the performance characteristics of a ducted propeller at zero angle of attack. The results show the opportunity to increase the propulsive efficiency in comparison to an open propeller provided that an optimisation of the duct shape is carried out.

However, several numerical methods have also appeared for the analysis of the flow around ducted propellers. For example, Gray and Wright [15] proposed a vortex wake model for the incompressible, inviscid, steady flow around a ducted fan. In their approach, the effects of the hub, of the tip clearance, and of the wake contraction were neglected. Guerrero et al. [16] developed a multidisciplinary optimisation code for the design and analysis of ducted fan vertical take-off and landing unmanned air vehicles (VTOL UAV). The duct forces prediction is based upon the empirical data furnished by Fletcher [2], while the propeller is modelled by means of two different models. The first one is a simple actuator disk model, while the second one is a blade/vortex element method. Chang and Rajagopalan [17] modelled the axisymmetric and incompressible flow around a ducted fan by means of an actuator disk approach. Specifically, the flow field is solved with the help of a classical CFD technique, while the rotor is represented by a set of sources in the momentum equation. The strength of these sources is iteratively evaluated through a blade element method. The results of the model were also validated against experimental data for the Trek Aerospace ducted fan and for the Micro-Craft's LADFUAV configurations. A similar approach was employed by Ahn and Lee [18] to investigate how the duct exit angle and the shape of the duct leading edge affect the performance of a ducted fan. Lind et al. [19] coupled a blade element method for the calculation of the propeller forces with a potential multiorder panel code for the evaluation of the flow field around a ducted fan. Experimental campaigns were also performed on a 10 in diameter and 2-bladed propeller surrounded by a 6 -in chord duct. The computational results showed a good agreement with experimental data for low and moderate values of the angle of attack. Kinnas et al. [20] analysed the flow around ducted propellers through a perturbation potential-based panel method which employs a full wake alignment scheme. The results are in good agreement both with experiments and CFD simulations.

In this paper the axisymmetric flow field around a ducted rotor with zero angle of attack is thoroughly analysed by

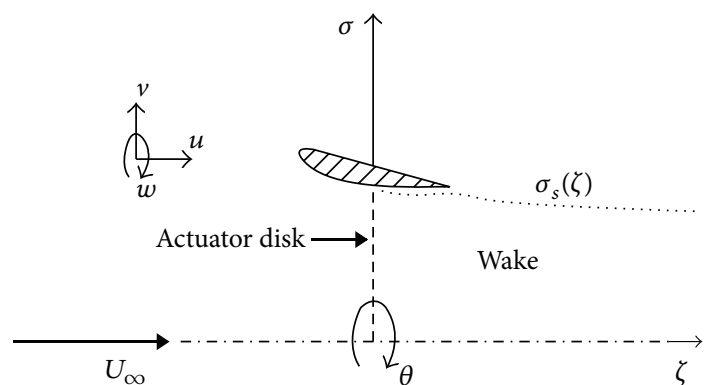

FIGURE 1: Schematic representation of the actuator disk flow problem.

means of a nonlinear and semi-analytical model [21-26] which is able to deal with some crucial aspects of shrouded systems like the interaction between the rotor and the duct and the slipstream contraction and rotation. The proposed procedure is characterised by a very low computational cost that makes it very appealing as analysis tool in the preliminary steps of a design procedure of hierarchical type. A verification of the correctness of the method through a comparison with CFD simulations has been carried out in [27] and [28] for a ducted propeller and a ducted turbine, respectively. With the help of this method, the effects of the thickness and camber of the duct on the performance coefficients of a ducted propeller will be analysed in detail with a focus on the propulsive efficiency.

\section{The Actuator Disk Flow Model}

Since its first appearance in the axial momentum theory of Rankine [29] and Froude [30], the actuator disk constitutes a widely diffused method for the analysis of the stationary, incompressible ( $\rho=$ const.), inviscid, and axisymmetric flow around open and ducted rotors. In the present paper, due to the axisymmetric flow assumption, a cylindrical coordinate system is introduced and the axial, radial, and tangential coordinates are named $\zeta, \sigma$, and $\theta$, respectively (see Figure 1 ). The actuator disk is a synthetic model which represents the effects of the rotor by introducing a jump in the static pressure $p$ and in the tangential velocity $w$ across a disk of infinitesimal thickness. Contrariwise, the radial $v$ and axial $u$ velocities are considered to be continuous functions in the whole flow field. By so doing, a discontinuity in the Bernoulli constant

$$
H=\frac{p}{\rho}+\frac{1}{2}\left(u^{2}+v^{2}+w^{2}\right)
$$

also appears, so that the mechanical energy $\mathscr{H}$ transferred to the fluid reads

$$
\mathscr{H}=\Delta H=\frac{\Delta p}{\rho}+\frac{\Delta w^{2}}{2},
$$

where $\Delta H, \Delta p$, and $\Delta w^{2} / 2$ are the jump across the disk in the Bernoulli constant, in the static pressure, and in the kinetic tangential energy, respectively. Having said that, the flow field can be divided into two regions as reported in Figure 1. 
The first one is the so called propeller wake which is defined as the space region $\left\{(\zeta, \sigma) \in \mathbb{R} \times \mathbb{R}: \zeta>0, \sigma<\sigma_{s}(\zeta)\right\}$ (see Figure 1). In the latter definition, the function $\sigma_{s}(\zeta)$, which describes the shape of the wake (see Figure 1), is not known in advance and must be evaluated as a part of the solution. Due to the energy transfer occurring through the rotor, $H$ is not constant in the wake. The second region is the domain outside the wake and it is characterised by a constant value of $H$ equal to $H_{\infty}=p_{\infty} / \rho+U_{\infty}^{2} / 2$, where $U_{\infty}$ and $p_{\infty}$ are the velocity and the static pressure of the free stream.

Following the approach suggested by $\mathrm{Wu}$ [31], the flow problem is formulated in terms of the Stokes stream function, which is defined as the function $\Psi$ satisfying the following relations:

$$
\begin{aligned}
& u=\frac{1}{\sigma} \frac{\partial \Psi}{\partial \sigma} \\
& v=-\frac{1}{\sigma} \frac{\partial \Psi}{\partial \zeta}
\end{aligned}
$$

Then, from the continuity and momentum equation, it can be proven [21] that the following differential problem holds for the through-flow around a ducted actuator disk:

$$
\begin{aligned}
\frac{\partial^{2} \widehat{\Psi}}{\partial \widehat{\sigma}^{2}}-\frac{1}{\widehat{\sigma}} \frac{\partial \widehat{\Psi}}{\partial \widehat{\sigma}}+\frac{\partial^{2} \widehat{\Psi}}{\partial \widehat{\zeta}^{2}} & =\delta\left(2-\frac{\widehat{\mathscr{H}} J^{2}}{\pi^{2}}\right) \frac{\mathrm{d} \widehat{\mathscr{H}}}{\mathrm{d} \widehat{\Psi}} \\
\frac{1}{2 \widehat{\sigma}} \frac{\partial \widehat{\Psi}}{\partial \widehat{\sigma}} & \longrightarrow 1
\end{aligned}
$$

$$
\begin{aligned}
& \text { as } \widehat{\zeta} \longrightarrow-\infty \text { or } \widehat{\sigma} \longrightarrow \infty \\
& \frac{\partial \widehat{\Psi}}{\partial \widehat{\zeta}} \longrightarrow 0 \quad \text { as } \widehat{\zeta} \longrightarrow \infty \text { or } \widehat{\sigma} \longrightarrow \infty \\
& \widehat{\Psi}=\text { const. } \quad \text { on } C,
\end{aligned}
$$

where $C$ is the contour of the duct cross section, $J=$ $U_{\infty} /\left(2 n \sigma_{\text {ad }}\right)$ is the advance coefficient of the propeller, $U_{\infty}$ is the free stream velocity, $n$ is the rotational speed of the rotor in revolutions per second and, finally, $\sigma_{\mathrm{ad}}$ is the actuator disk radius. In problem (4)-(7), the following dimensionless quantities have also been employed:

$$
\begin{aligned}
\widehat{\sigma} & =\frac{\sigma}{\sigma_{\mathrm{ad}}} \\
\widehat{\zeta} & =\frac{\zeta}{\sigma_{\mathrm{ad}}} \\
\widehat{\Psi} & =\frac{2 \Psi}{U_{\infty} \sigma_{\mathrm{ad}}^{2}}, \\
\widehat{\mathscr{H}} & =\frac{2 \mathscr{H}}{U_{\infty}^{2}} .
\end{aligned}
$$

Function $\delta$, appearing at the right hand-side of (4), is equal to 1 inside the wake and zero otherwise. As stated before, the slipstream edge $\sigma_{s}(\zeta)$, defining the space region outside of which $\delta$ vanishes, is not known beforehand and it must be computed as a part of the solution. The linear elliptic operator appearing at the left hand side of (4) differs from the Laplacian operator due to the presence of the minus sign. The right hand-side of the same equation is a function of the two physical quantities $J$ and $\widehat{\mathscr{H}}$ which have to be prescribed in advance. This means that $J$ and $\widehat{\mathscr{H}}$ have to be regarded as input quantities to the method. With the help of the Stokes stream function definition (3) the meaning of the conditions at infinity (5) and (6) can be easily understood. In fact, the first one requires that, outside the wake, the dimensionless axial velocity $\widehat{u}=u / U_{\infty}$ tends to one at infinity. Then, in order to comply with condition (6), the dimensionless radial velocity $\widehat{v}=v / U_{\infty}$ has to vanish everywhere at infinity. The last requirement ( 7 ) is the duct impermeability condition.

Once the through-flow problem (4)-(7) has been solved, the tangential component of the velocity can be easily evaluated by means of the angular momentum or Euler equation:

$$
\widehat{\mathscr{H}}=\frac{2 \pi \widehat{\sigma} \widehat{w}}{J} .
$$

With the help of the Hankel transform it can be proven that the solution of problem (4)-(7) can be regarded as the superposition of the flow induced by a set of ring vortices (see [21] for mathematical details). The ring vortex is one of the classical vorticity singularities which is often employed to represent axisymmetric flows [32]. Considering a single ring vortex of radius $r$, unity strength, and located at $(\zeta=z$, $\sigma=0)$, the induced stream function and velocities are $[33,34]$

$$
\begin{aligned}
& \Psi_{r v}=\frac{r}{2} \int_{0}^{\infty} e^{-s|\zeta-z|} \mathrm{J}_{1}(s r) \mathrm{J}_{1}(s \sigma) \mathrm{d} s \\
& u_{r v}=\frac{r}{2} \int_{0}^{\infty} e^{-s|\zeta-z|} s J_{1}(s r) \mathrm{J}_{0}(s \sigma) \mathrm{d} s \\
& v_{r v}= \pm \frac{r}{2} \int_{0}^{\infty} e^{-s|\zeta-z|} s \mathrm{~J}_{1}(s r) \mathrm{J}_{1}(s \sigma) \mathrm{d} s
\end{aligned}
$$

where $\mathrm{J}_{1}$ and $\mathrm{J}_{0}$ are the first kind Bessel functions of the first and zero order, respectively. In more details, two ring vortex systems are adopted to represent the flow around a ducted rotor. The first one is the actuator disk ring vortex system which models the flow induced by the propeller. The second one is the duct ring vortex system and it models the flow induced by the duct. Moreover, two density strength distributions $\gamma_{\mathrm{ad}}(\zeta, \sigma)$ and $\gamma_{d}(\zeta, \sigma)$ can be introduced for the actuator disk and the duct ring vortices, respectively. Consequently, the exact solution $\Psi$ can be cast in the following form:

$$
\begin{aligned}
\Psi & =\frac{U_{\infty} \sigma^{2}}{2}+\frac{\sigma}{2} \\
& \cdot \int_{0}^{\infty} \int_{0}^{\sigma_{s}(\zeta)} \int_{0}^{\infty} e^{-s|\zeta-z|} \gamma_{\mathrm{ad}} r \mathrm{~J}_{1}(s r) \mathrm{J}_{1}(s \sigma) \mathrm{d} s \mathrm{~d} r \mathrm{~d} z \\
& +\frac{\sigma}{2} \oint_{C} \int_{0}^{\infty} e^{-s|\zeta-z|} \gamma_{d} r(c) \mathrm{J}_{1}(s r) \mathrm{J}_{1}(s \sigma) \mathrm{d} s \mathrm{~d} c,
\end{aligned}
$$

where $c$ is the curvilinear abscissa along the contour $C$ of the duct cross section. The first term appearing at the right 
hand-side of the above equation is the Stokes stream function of the free stream. The second addendum is the Stokes stream function induced by the actuator disk ring vortex system. Finally, the last integral represents the Stokes stream function induced by the duct. It is noteworthy that the second and third addendum can be simply obtained by integrating (10) over the wake and the contour $C$, respectively.

The density strengths $\gamma_{\text {ad }}(\zeta, \sigma)$ and $\gamma_{d}(\zeta, \sigma)$ have to be evaluated in such a way that the resulting overall solutions (13) satisfy both (4) and the boundary conditions (5)-(7). To this aim, the density strength $\gamma_{\mathrm{ad}}$ has to be related to the advance coefficient $J$ and to $\mathscr{H}$ through the following equation [21]:

$$
\gamma_{\mathrm{ad}}(\zeta, \sigma)=\delta\left(2-\frac{\widehat{H} J^{2}}{\pi^{2}}\right) \frac{\mathrm{d} \widehat{H}}{\mathrm{~d} \widehat{\Psi}} .
$$

Furthermore, the density strength $\gamma_{d}(c)$ has to satisfy the following Fredholm second kind integral equation [21]:

$$
\begin{aligned}
& \frac{1}{2} \gamma_{d}(c)+\oint_{C} k(c, \widetilde{c}) \gamma_{d}(\widetilde{c}) \mathrm{d} \widetilde{c} \\
& \quad=\left(U_{\infty}+u_{\mathrm{ad}}(c)\right) \cos \beta(c)+v_{\mathrm{ad}}(c) \sin \beta(c),
\end{aligned}
$$

where $\beta(c)$ is the local profile slope, $k(c, \widetilde{c})$ is the velocity parallel to the surface at $c$, induced by a ring vortex of unit strength located at $\widetilde{c}$, and $u_{\text {ad }}(c)$ and $v_{\text {ad }}(c)$ are the axial and radial velocities at $c$ induced by the actuator disk ring vortex system, respectively. Equation (15) is the homogeneous Dirichlet boundary condition on the tangent to the wall velocity just beneath the duct vorticity sheet of ring vortices, and, moreover, it is equivalent to the impermeability condition on the duct surface [35].

It should be noted that the solution $\Psi(\zeta, \sigma)$ cannot be directly evaluated through (13) since all quantities $\gamma_{\mathrm{ad}}, \gamma_{d}$, and $\sigma_{s}$ depend upon $\Psi$. This means that (13) is the exact but implicit solution of the flow around a ducted actuator disk. In order to make (13) explicit, a semi-analytical and iterative procedure has been developed in [21], but it will be not be reported hereafter for the sake of brevity. Once the solution $\Psi(\zeta, \sigma)$ of (13) has been obtained, the whole velocity and pressure flow field can be easily computed. The aforementioned iterative procedure can handle load distributions expressed as an algebraic polynomial of the Stokes stream function:

$$
\mathscr{H}(\Psi)=\sum_{m=0}^{M} a_{m}\left(\frac{\Psi}{\Psi_{\mathrm{ad}}}\right)^{m} .
$$

In the above equation, $\Psi_{\text {ad }}$ is the stream function at the apex of the disk, while $a_{m}$ are input parameters to be supplied to the procedure. In the following, a parabolic load distribution $(M=2)$ will be employed for all simulations, while the advance coefficient $J$ will be set equal to 0.5 .
Moreover, the following classical definition for the thrust and power coefficients will be adopted:

$$
\begin{aligned}
C_{T} & =\frac{T}{(1 / 2) \rho U_{\infty}^{2} \pi \sigma_{\mathrm{ad}}^{2}}, \\
C_{P} & =\frac{P}{(1 / 2) \rho U_{\infty}^{3} \pi \sigma_{\mathrm{ad}}^{2}} .
\end{aligned}
$$

As customary, $T$ and $P$ are the thrust and the power experienced by the device, respectively. The overall thrust $T$ can also be regarded as the algebraic sum of the rotor $\left(T_{\text {rot }}\right)$ and the duct $\left(T_{\text {duct }}\right)$ thrust, so that the following equation holds:

$$
C_{T}=C_{T, \text { rot }}+C_{T, \text { duct }}
$$

where $C_{T, \text { rot }}=2 T_{\text {rot }} / \rho U_{\infty}^{2} \pi \sigma_{\text {ad }}^{2}$ and $C_{T \text {,duct }}=2 T_{\text {duct }} /$ $\rho U_{\infty}^{2} \pi \sigma_{\text {ad }}^{2}$. By convention, the duct thrust coefficient is considered to be negative if $T_{\text {duct }}$ is discordant with $T_{\text {rot }}$ and vice-versa. Finally, the ideal propulsive efficiency is evaluated through the standard relation

$$
\eta_{i}=\frac{C_{T}}{C_{P}}
$$

The aforementioned performance coefficient can be easily evaluated through the proposed semi-analytical method. In particular, since the elemental rotor thrust $\mathrm{d} T_{\text {rot }}$ is defined as the product between the static pressure jump across the disk $\Delta p$ and the infinitesimal area $2 \pi \sigma \mathrm{d} \sigma$, it is easy to prove that the rotor thrust coefficient can be expressed as [21]

$$
C_{T, \text { rot }}=2 \int_{0}^{1} \Delta \widehat{p} \widehat{\sigma} \mathrm{d} \widehat{\sigma}
$$

where $\Delta \widehat{p}=2 \Delta p /\left(\rho U_{\infty}^{2}\right)$ can be evaluated from (2) once the tangential velocity has been obtained from (9). The duct thrust can be evaluated by integrating the static pressure over the duct surface, so that $C_{T, \text { duct }}$ reads $[21,36]$

$$
\begin{aligned}
C_{T, \text { duct }} & =\frac{2}{\rho U_{\infty}^{2} \pi \sigma_{\mathrm{ad}}^{2}} \oint_{C} p \mathbf{i}_{\zeta} \cdot \mathbf{n} 2 \pi \sigma \mathrm{d} c \\
& =2 \oint_{C} C_{p, \text { wall }} \widehat{\sigma} \mathbf{i}_{\zeta} \cdot \mathbf{n} \mathrm{d} \widehat{c},
\end{aligned}
$$

where $C_{p \text {,wall }}=2\left(p-p_{\infty}\right) /\left(\rho U_{\infty}^{2}\right)$ is the wall pressure coefficient, $\widehat{c}=c / \sigma_{\text {ad }}$ is the dimensionless curvilinear abscissa, $\mathbf{n}$ is the outward pointing normal to the duct surface, and $\mathbf{i}_{\zeta}$ is the unit vector of the $\zeta$ axis. Finally, the power absorbed in the infinitesimal rotor area $2 \pi \sigma \mathrm{d} \sigma$ is defined as the product between the elemental mass flow $\rho u(0, \sigma) 2 \pi \sigma \mathrm{d} \sigma$ and the mechanical energy transferred $\mathscr{H}$. Then, the power coefficient can be cast in the following form [21]:

$$
\begin{aligned}
C_{P} & =\frac{2 \int_{0}^{\sigma_{\mathrm{ad}}} \mathscr{H} \rho u(0, \sigma) 2 \pi \sigma \mathrm{d} \sigma}{\rho U_{\infty}^{3} \pi \sigma_{\mathrm{ad}}^{2}} \\
& =2 \int_{0}^{1} \widehat{\mathscr{H}} \hat{u}(0, \widehat{\sigma}) \hat{\sigma} \mathrm{d} \widehat{\sigma} .
\end{aligned}
$$




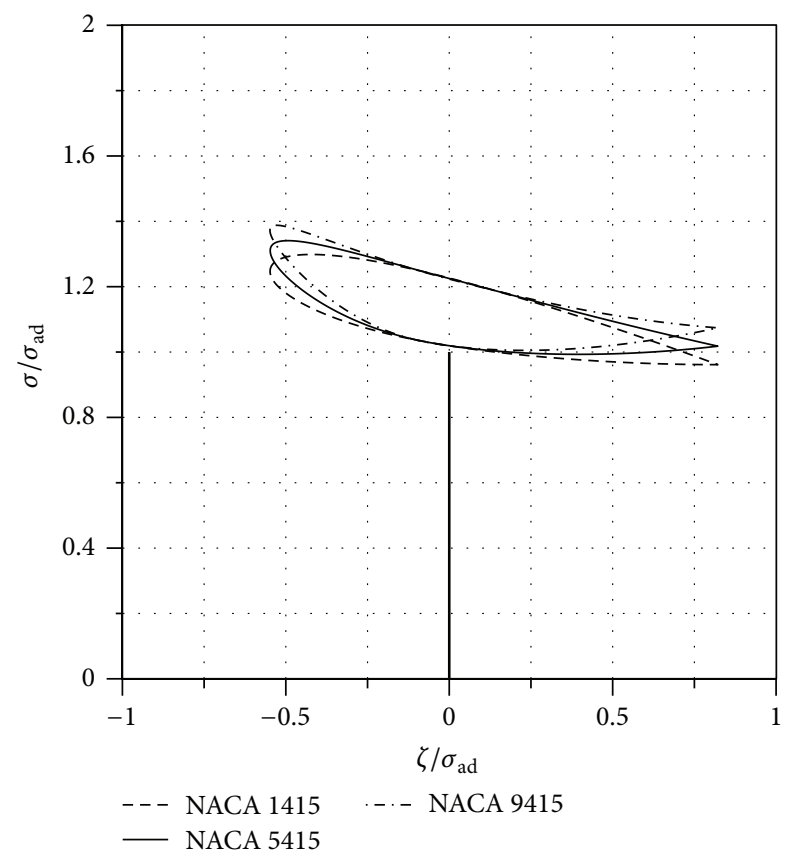

FIgURE 2: Profiles employed to investigate the effects of the duct cross section camber.

TABLE 1: Geometrical details of the reference profile.

\begin{tabular}{lc}
\hline Ratio between the tip gap and $\sigma_{\mathrm{ad}}$ & $\sim 2 \%$ \\
Ratio between the chord and $\sigma_{\mathrm{ad}}$ & 1.4 \\
Angle between the chord and the $\zeta$ axis & $12.0^{\circ}$ \\
\hline
\end{tabular}

\section{Results}

In this section, the effects of the geometry of the duct on the performance coefficients of a ducted propeller will be described with a focus on the propulsive efficiency. In particular, an analysis of the effects of a finite variation of the thickness and camber of the duct will be conducted with the help of the semi-analytical actuator disk method described in the previous section. The scope of the analysis is twofold. Firstly, some information about the role played by the duct and about the operating principles of this kind of machinery are collected. Then, some broad guidelines for the design of ducted propellers are outlined. The analysis relies on the use of the NACA 4 digits wing sections. In the present study, a NACA 5415 is adopted as reference profile (see Table 1 for geometrical details).

Then, the effects of the camber are investigated by changing the first of the four digits; namely, a NACA 1415 and a NACA 9415 have been employed (see Figure 2). In fact, in the NACA 4-digit numbering system the first digit describes the maximum value of the mean line ordinate in percentage of the chord, that is, the maximum camber. Instead, the effects of the thickness are investigated by means of a NACA 5405 and a NACA 5425 profile (see Figure 3). In fact, as well-known, the last two digits denote the maximum thickness as percentage of the chord.

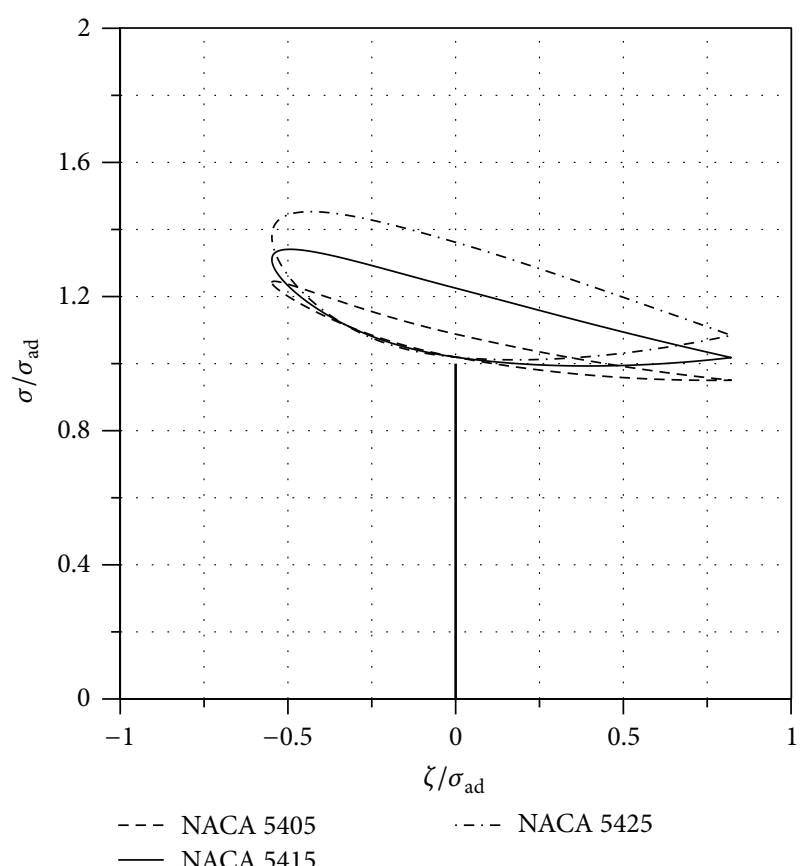

FIGURE 3: Profiles employed to investigate the effects of the duct cross section thickness.

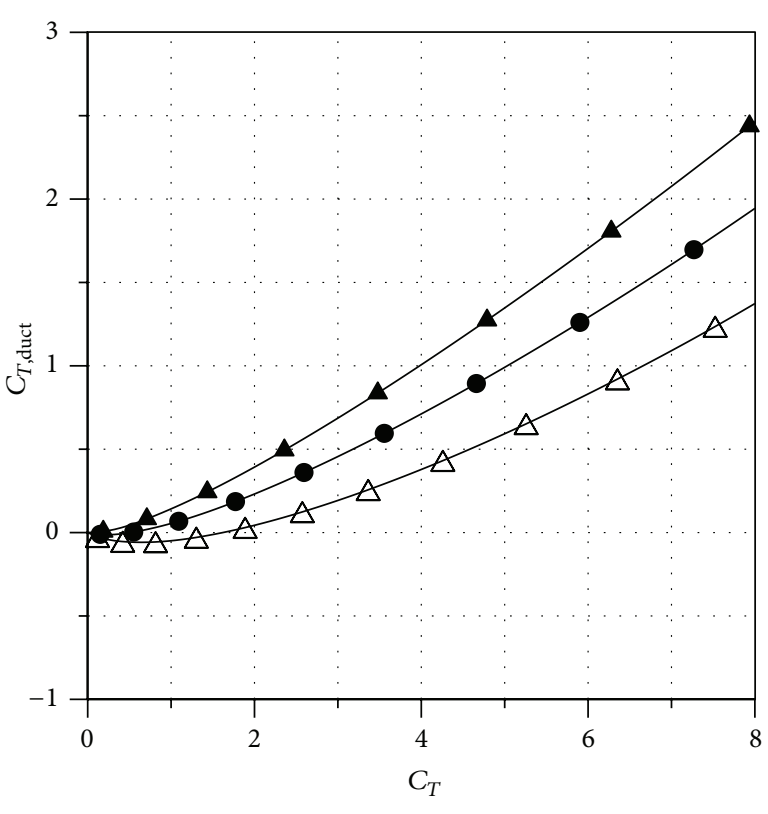

$\triangle$ NACA 5405

- NACA 5425

Figure 4: Effects of the profile thickness on the duct thrust coefficient.

The effect of the profile thickness on the duct thrust coefficient, on the mass flow swallowed by the rotor, and, finally, on the propulsive efficiency is reported in Figures 4,5 , and 6 , respectively. In the aforementioned figures, the dashed lines represent the results of the open rotor configuration obtained through a modified version of the 


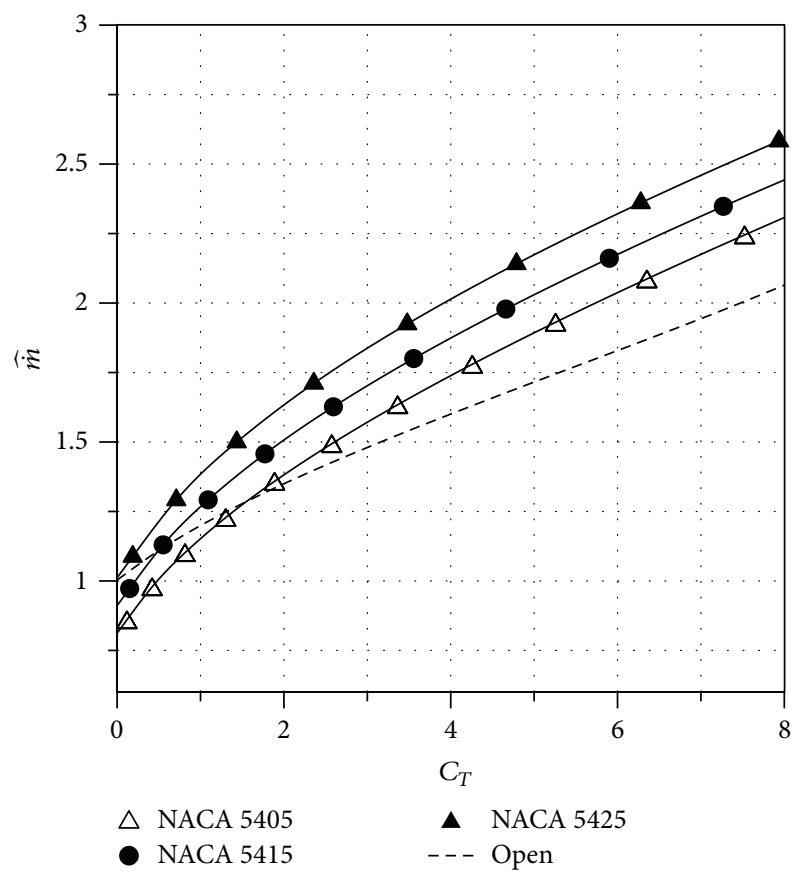

FIGURE 5: Effects of the profile thickness on the mass flow swallowed by the rotor.

semi-analytical actuator disk method which does not take into account the presence of the duct. According to the wellknown axial momentum theory, a negative value of the duct thrust coefficient (see Figure 4) returns a decrease in the dimensionless mass flow $\left(\widehat{\dot{m}}=\dot{m} /\left(\rho U_{\infty} \pi \sigma_{\text {ad }}^{2}\right)\right)$ swallowed by the rotor in comparison to an open propeller with the same $C_{T}$ (see Figure 5). Moreover, a negative value of $C_{T \text {,duct }}$ also induces a decrease in $\eta_{i}$ in comparison to an open propeller (see Figure 6). In particular, for lower values of $C_{T}$, ducts NACA 5405 and NACA 5415 are characterised by a negative duct thrust and, consequently, by a reduced value of $\hat{\dot{m}}$ and $\eta_{i}$. Contrariwise, for higher $C_{T}$ values both ducts are characterised by a positive $C_{T \text {,duct }}$ and therefore by an increased value of the propulsive efficiency in comparison to an open propeller. In turn, the NACA 5425 profile always experiences a positive duct thrust and an enhancement in the propulsive efficiency.

Some insights on the behaviour of $C_{T, \text { duct }}$ when $C_{T}$ is decreased can be obtained through a closer look at the wall pressure coefficient distributions. In particular, Figure 7 represents $C_{p \text {,wall }}$ as a function of $\zeta / \sigma_{\text {ad }}$ for the duct NACA 1405 and for three different $C_{T}$ values. As already stressed, the actuator disk is placed at $\zeta / \sigma_{\text {ad }}=0$.

It can be observed that, for a high value of $C_{T}$, the front stagnation point is located on the external surface of the duct. This means that a large acceleration and, consequently, a very negative value of $C_{p \text {,wall }}$ exists at the front part of the duct inner surface. Since $\mathbf{i}_{\zeta} \cdot \mathbf{n}$ is also negative in this last part of the duct, a huge positive contribution to the overall integral value of $C_{T \text {,duct }}$ (see (21)) is due to the front part of the inner duct surface. Looking at Figure 7, it is also easy to realise that

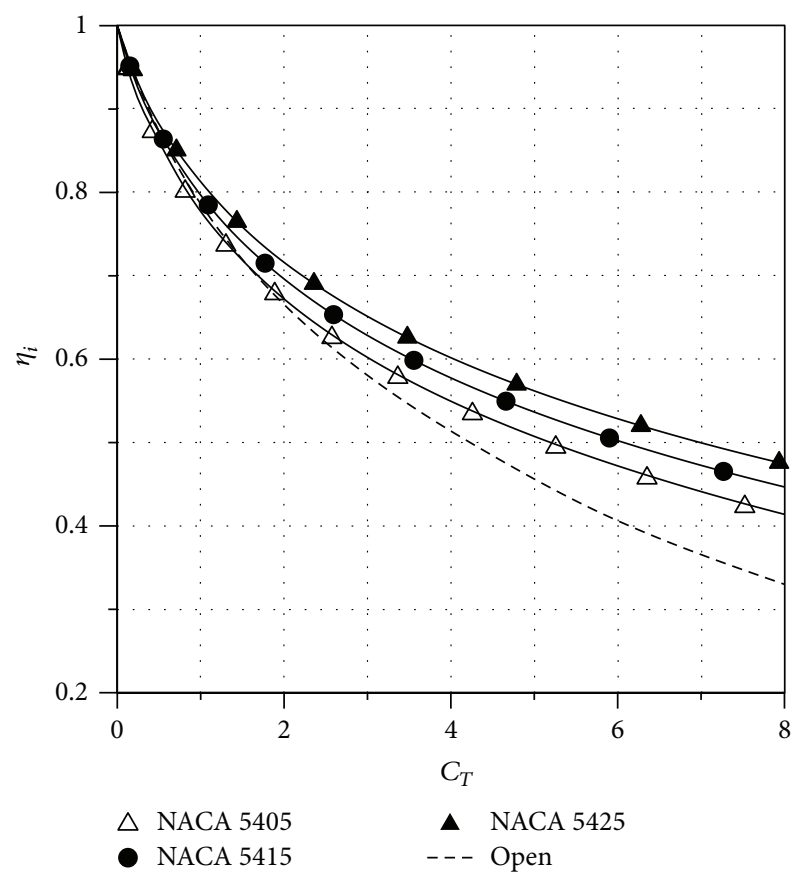

FIGURE 6: Effects of the profile thickness on the propulsive efficiency.

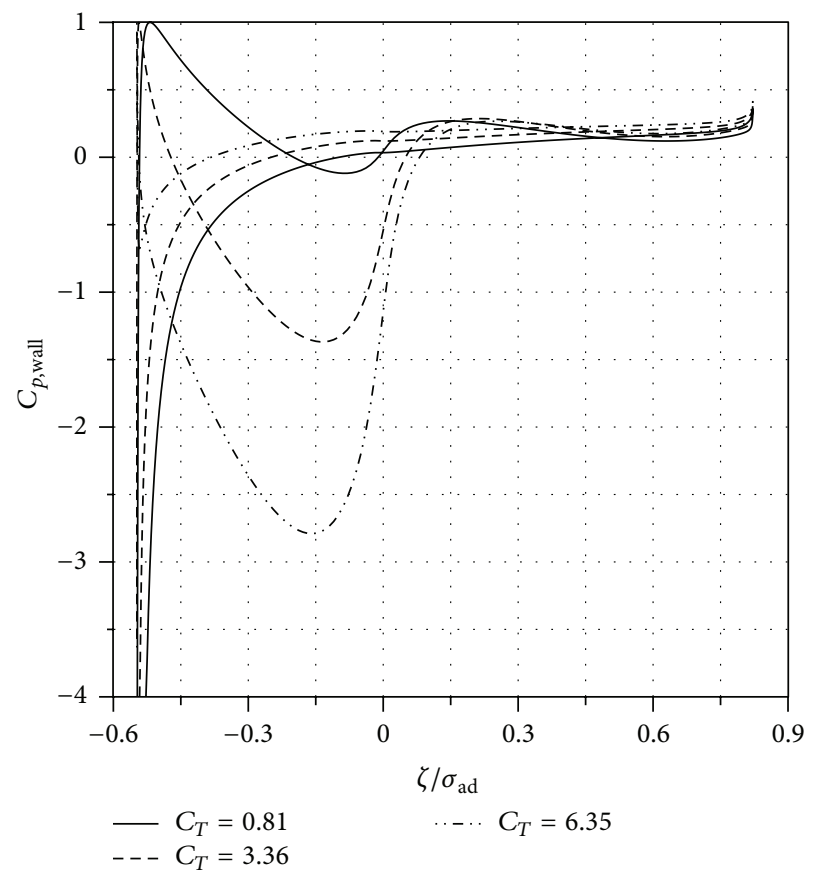

FIGURE 7: Wall pressure coefficient distribution along the duct NACA 5405.

the most important contribution to $C_{T, \text { duct }}$ is the one due to the front part of the inner surface. When $C_{T}$ is decreased, the mass flow swallowed by the propeller is decreased too. Consequently, the front stagnation point moves towards the inner part of the duct so that $C_{p \text {,wall }}$ tends to become less negative in the front part of the inner surface and $C_{T \text {,duct }}$ tends 


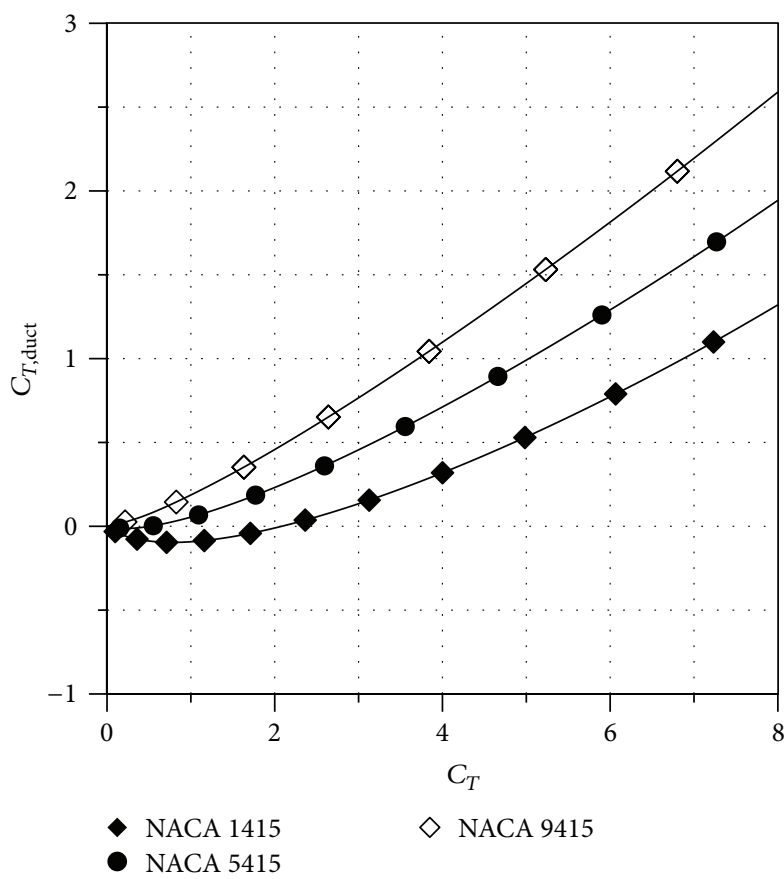

Figure 8: Effects of the profile camber on the duct thrust coefficient.

to become less positive or even negative. The effects of the front stagnation point displacement with $C_{T}$ are mitigated when the thickness of the profile is increased. In fact, in a thicker profile, the profile curvature at the leading edge is decreased as well as the acceleration needed to go around it.

To summarise, an increase in the duct thickness yields a beneficial effect on the propulsive efficiency (see Figure 6). However, compared with the open rotor configuration, thin profiles only preserve this advantage for sufficiently high values of the thrust coefficient.

Turning now to the camber effect, Figure 8 shows the duct thrust coefficient $C_{T \text {,duct }}$ as a function of the overall thrust coefficient $C_{T}$ for three different profiles. Once again, if $C_{T \text {,duct }}$ is negative, then the rotor swallows a lower mass flow in comparison to the open configuration (see Figure 9) and consequently a lower value of the propulsive efficiency is achieved (see Figure 10). This is the case of the NACA 1415 and 5415 profiles, which experience a negative $C_{T \text {,duct }}$ for low values of $C_{T}$. Conversely, at the higher value of $C_{T}$, the ducted configuration always induces an enhancement of $\eta_{i}$ for all profiles. The reasons behind this behavior are again related to the displacement of the position of the front stagnation point with the thrust coefficient; as it can be seen in Figure 11 a propeller ducted with the most cambered NACA 9415 profile behaves differently, since it is characterised by an enhanced propulsive efficiency in the whole range of $C_{T}$. To summarise, an increase in the duct camber yields a beneficial effect on the propulsive efficiency (see Figure 10). However, compared with the open rotor configuration, poorly cambered profiles only preserve this advantage for sufficiently high values of the thrust coefficient $C_{T}$.

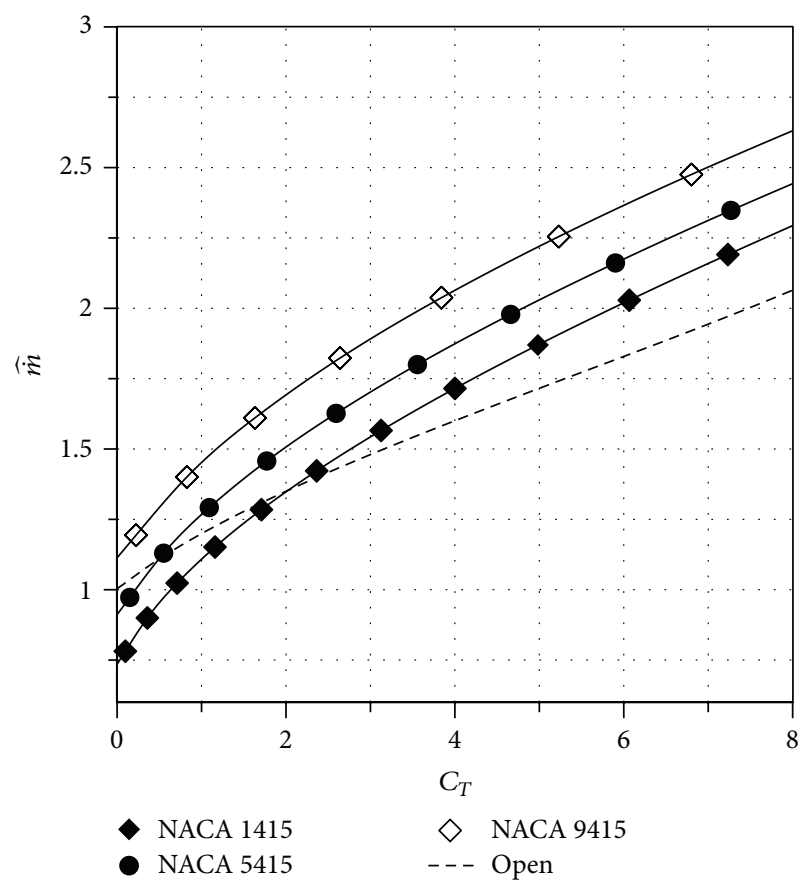

FIGURE 9: Effects of the profile camber on the mass flow swallowed by the rotor.

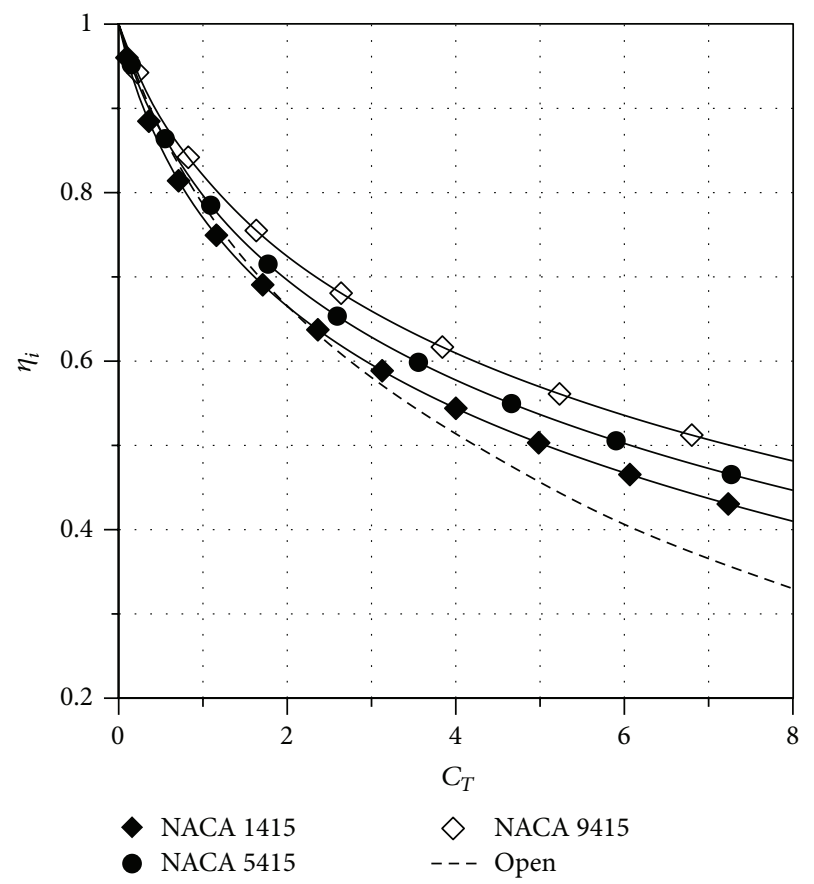

FIGURE 10: Effects of the profile camber on the propulsive efficiency.

\section{Conclusions}

In the paper, the axisymmetric flow field around a ducted propeller/fan has been thoroughly analysed by means of a nonlinear and semi-analytical model which is able to deal with some crucial aspects of shrouded systems like the interaction between the rotor and the duct, and the slipstream 


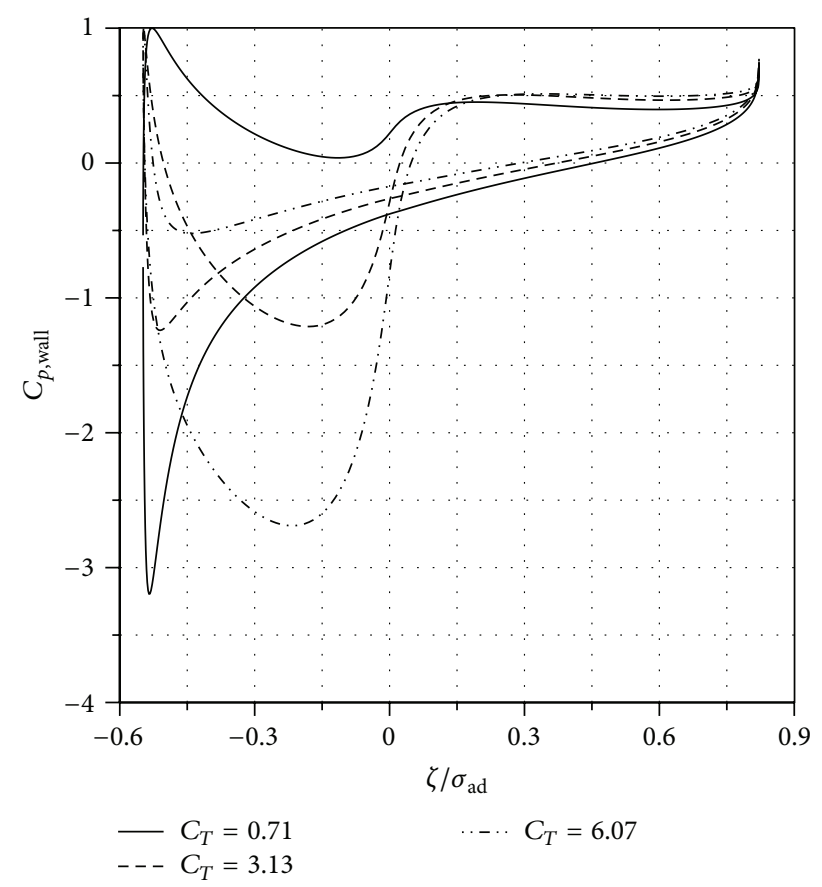

FIGURE 11: Wall pressure coefficient distribution along the duct NACA 1415.

contraction and rotation. According to the axial momentum theory, it has been found that if the duct thrust is concordant with the rotor propulsive thrust, then the duct induces a gain in the mass flow swallowed by the rotor and in the propulsive efficiency. Contrariwise, if the duct thrust is discordant with the rotor thrust, then the duct induces a decrease in the swallowed mass flow and in the propulsive efficiency. The effects of the thickness and of the camber of the duct on the performance coefficients of a ducted propeller have also been investigated. In particular, it has been found that an augmentation of the camber and of the thickness has a beneficial effect on the propulsive efficiency. Moreover, a low value of the camber and of the thickness can even bring about a decrease of $\eta_{i}$, especially at low values of $C_{T}$.

\section{Conflict of Interests}

The authors declare that there is no conflict of interests regarding the publication of this paper.

\section{References}

[1] W. Kruger, "On wind tunnel tests and computations concerning the problem of shrouded propellers," NACA TN no. 1202, 1949.

[2] H. S. Fletcher, "Experimental investigation of lift, drag, and pitching moment of five annular airfoils," NACA TN 4117, 1957.

[3] K. W. Mort and P. F. Yaggy, "Aerodynamic characteristics of a 4 -foot-diameter ducted fan mounted on the tip of a semispan wing," NASA TN D-1301, 1962.

[4] K. W. Mort and B. Gamse, "A wind-tunnel investigation of a 7foot-diameter ducted propeller," Tech. Rep. NASA-TN-D-4142, 1967.
[5] D. M. Black, H. S. Wainauski, and C. Rohrbach, "Shrouded propellers-a comprehensive performance study," in Proceedings of the AIAA 5th Annual Meeting and Technical Display, Philadelphia, Pa, USA, 1968.

[6] C. Young, "An investigation of annular aerofoils for turbofan engine cowls," ARC R\&M 3688, Her Majesty's Stationery Office, London, UK, 1972.

[7] A. I. Abrego and R. W. Bulaga, "Performance study of a ducted fan system," in Proceedings of the American Helicopter Society Aerodynamics, Acoustics, and Test and Evaluation Technical Specialists Meeting, San Francisco, Calif, USA, January 2002.

[8] G. F. Oweis, D. Fry, C. J. Chesnakas, S. D. Jessup, and S. L. Ceccio, "Development of a tip-leakage flow-part 1: the flow over a range of Reynolds numbers," Journal of Fluids Engineering, vol. 128, no. 4, pp. 751-764, 2006.

[9] G. F. Oweis, D. Fry, C. J. Chesnakas, S. D. Jessup, and S. L. Ceccio, "Development of a tip-leakage flow-part 2: comparison between the ducted and un-ducted rotor," Journal of Fluids Engineering, vol. 128, no. 4, pp. 765-773, 2006.

[10] W. Graf, J. Fleming, and W. Ng, "Improving ducted fan UAV aerodynamics in forward flight," in Proceedings of the 46th AIAA Aerospace Sciences Meeting and Exhibit, Reno, Nev, USA, January 2008.

[11] A. Akturk, A. Shavalikul, and C. Camci, "PIV measurements and computational study of a 5-inch ducted fan for V/STOL UAV applications," in Proceedings of the 47th AIAA Aerospace Sciences Meeting and Exhibit, Orlando, Fla, USA, January 2009.

[12] A. Akturk and C. Camci, "Experimental and computational assessment of a ducted-fan rotor flow model," Journal of Aircraft, vol. 49, no. 3, pp. 885-897, 2012.

[13] S. T. Koc, S. Yilmaz, D. Erdem, and M. S. Kavsaouglu, "Experimental investigation of a ducted propeller," in Proceedings of the 4th European Conference for Aerospace Sciences (EUCASS '11), 2011.

[14] S. Yilmaz, D. Erdem, and M. Kavsaoglu, "Performance of a ducted propeller designed for UAV applications at zero angle of attack flight: an experimental study," Aerospace Science and Technology, vol. 45, pp. 376-386, 2015.

[15] R. B. Gray and T. Wright, "A vortex wake model for optimum heavily loaded ducted fans," Journal of Aircraft, vol. 7, no. 2, pp. 152-158, 1970.

[16] I. Guerrero, K. Londenberg, P. Gelhausen, and A. Myklebust, "A powered lift aerodynamic analysis for the design of ducted fan uavs," in Proceedings of the 2nd AIAA Unmanned Unlimited Conference and Workshop and Exhibit, San Diego, Calif, USA, September 2003.

[17] I.-C. Chang and R. G. Rajagopalan, "CFD analysis for ducted fans with validation," in Proceedings of the 21st AIAA Applied Aerodynamics Conference, Orlando, Fla, USA, June 2003.

[18] J. Ahn and K. Lee, "A computational method for the performance modeling and design of a ducted fan system," in Proceedings of the 3rd Asian Simulation Conference, Jeju Island, Republic of Korea, 2004.

[19] R. Lind, J. K. Nathman, and I. Gilchrist, "Ducted rotor performance calculations and comparisons with experimental data," in Proceedings of the 44th AIAA Aerospace Sciences Meeting and Exhibit, AIAA, Reno, Nev, USA, January 2006.

[20] S. A. Kinnas, H. Fan, and Y. Tian, "A panel method with a full wake alignment model for the prediction of the performance of ducted propellers," Journal of Ship Research, vol. 59, no. 4, pp. 246-257, 2015. 
[21] R. Bontempo and M. Manna, "Solution of the flow over a nonuniform heavily loaded ducted actuator disk," Journal of Fluid Mechanics, vol. 728, pp. 163-195, 2013.

[22] R. Bontempo, The nonlinear actuator disk as applied to open and ducted rotors [Ph.D. thesis], University of Naples Federico II, Naples, Italy, 2014.

[23] R. Bontempo, M. Cardone, M. Manna, and G. Vorraro, "Ducted propeller flow analysis by means of a generalized actuator disk model," Energy Procedia, vol. 45, pp. 1107-1115, 2014.

[24] R. Bontempo, M. Cardone, M. Manna, and G. Vorraro, "A comparison of nonlinear actuator disk methods for the performance analysis of ducted propellers," in Proceedings of the 11th European Conference on Turbomachinery Fluid Dynamics and Thermodynamics (ETC '15), Madrid, Spain, March 2015.

[25] R. Bontempo, M. Cardone, and M. Manna, "Performance analysis of ducted marine propellers. Part I-decelerating duct," Applied Ocean Research, 2015.

[26] R. Bontempo and M. Manna, "Effects of the duct thrust on the performance of ducted wind turbines," Energy, In press.

[27] R. Bontempo, M. Cardone, M. Manna, and G. Vorraro, "A comparison of nonlinear actuator disk methods for the performance analysis of ducted marine propellers," Proceedings of the Institution of Mechanical Engineers Part A: Journal of Power and Energy, vol. 229, no. 5, pp. 539-548, 2015.

[28] R. Bontempo and M. Manna, "Performance analysis of open and ducted wind turbines," Applied Energy, vol. 136, pp. 405416, 2014.

[29] W. J. M. Rankine, "On the mechanical principles of the action of propellers," Transactions of the Institution of Naval Architects, vol. 6, pp. 13-39, 1865.

[30] R. E. Froude, "On the part played in propulsion by differences of fluid pressure," Transactions of the Institute of Naval Architects, vol. 30, pp. 390-405, 1889.

[31] T. Y. Wu, "Flow through a heavily loaded actuator disc," Schiffstechnik, vol. 9, pp. 134-138, 1962.

[32] G. K. Batchelor, An Introduction to Fluid Dynamics, Cambridge University Press, 1967.

[33] H. Lamb, Hydrodynamics, Cambridge University Press, 1932.

[34] A. B. Basset, A Treatise on Hydrodynamics, vol. 2, Deighton, Bell and Co, Cambridge, UK, 1888.

[35] R. I. Lewis, Vortex Element Methods for Fluid Dynamic Analysis of Engineering Systems, Cambridge University Press, Cambridge, UK, 1991.

[36] R. Bontempo and M. Manna, "A nonlinear and semi-analytical actuator disk method accounting for general hub shapes: part I-open rotor," Journal of Fluid Mechanics, In press. 


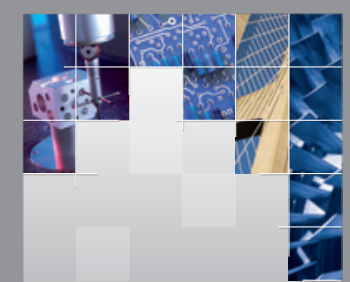

\section{Enfincering}
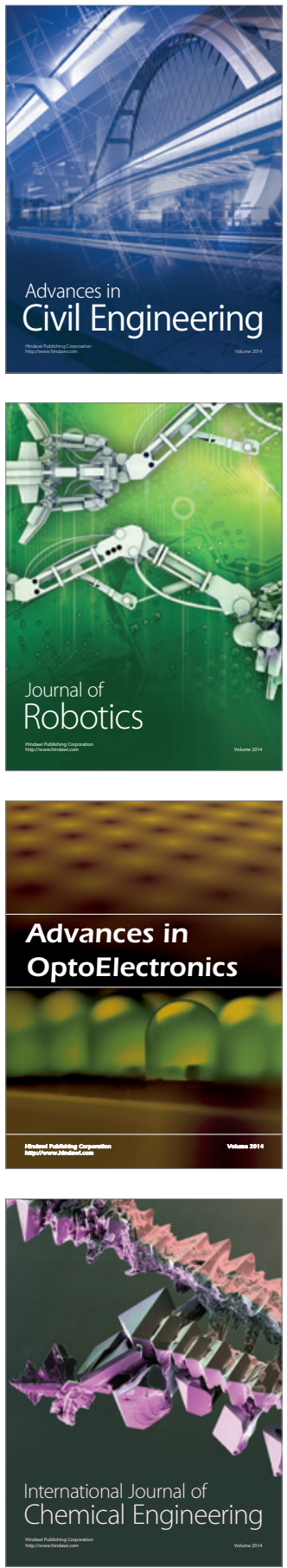

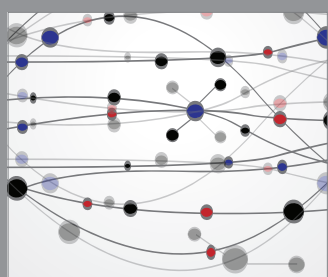

The Scientific World Journal

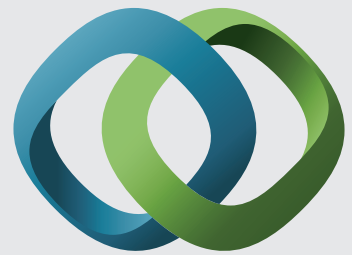

\section{Hindawi}

Submit your manuscripts at

http://www.hindawi.com
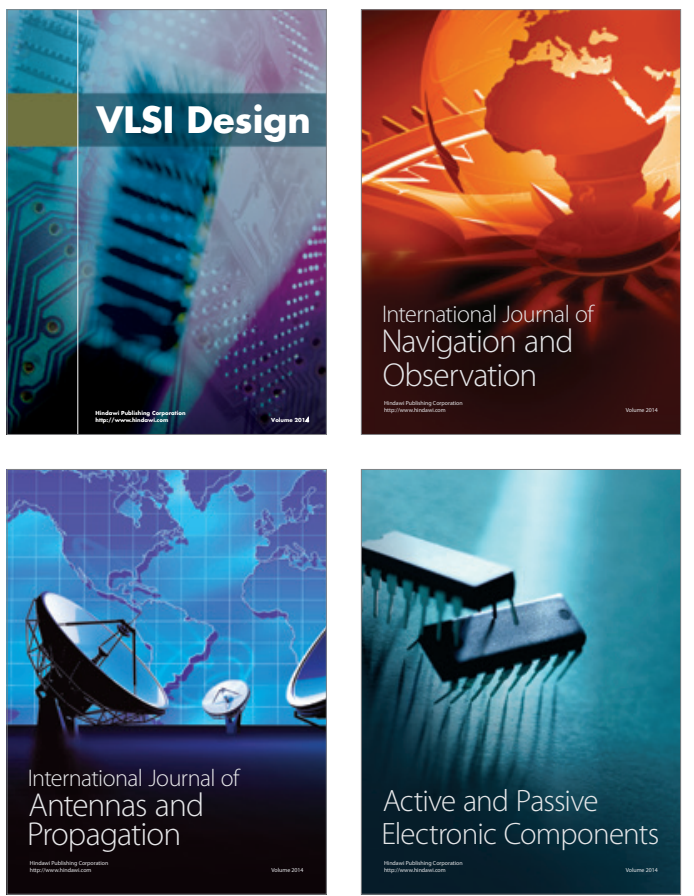
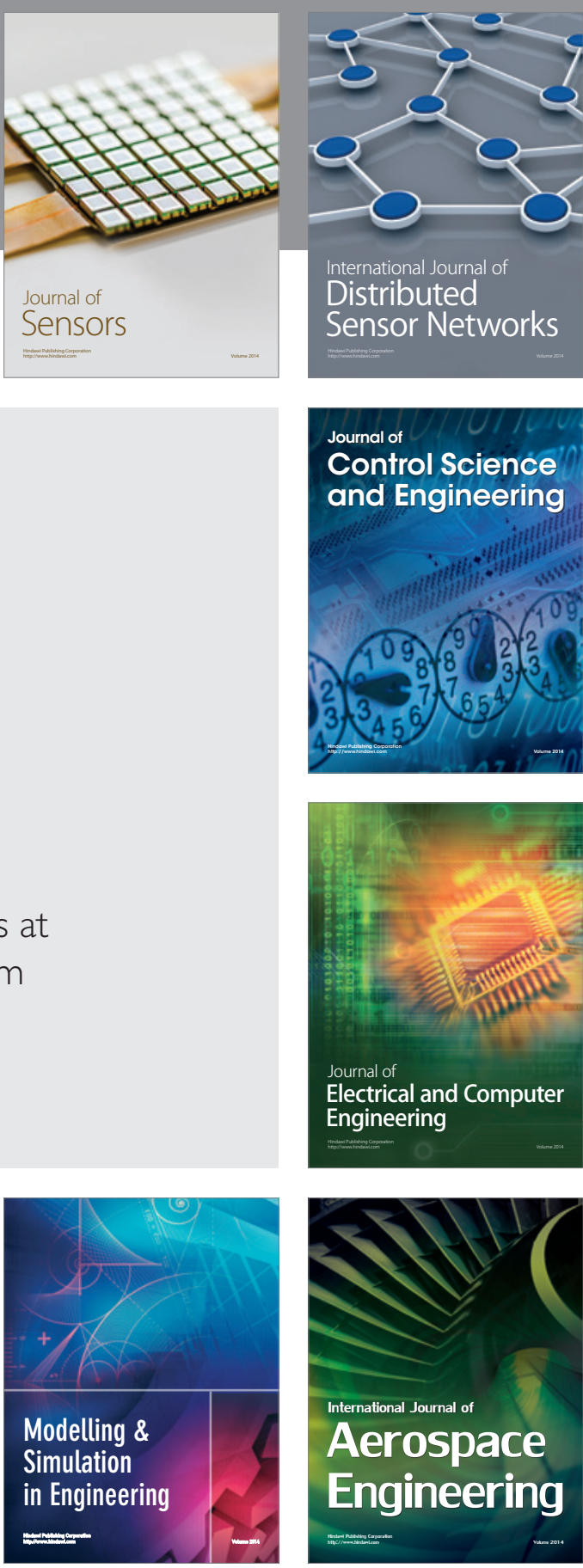

International Journal of

Distributed

Sensor Networks

Journal of

Control Science

and Engineering
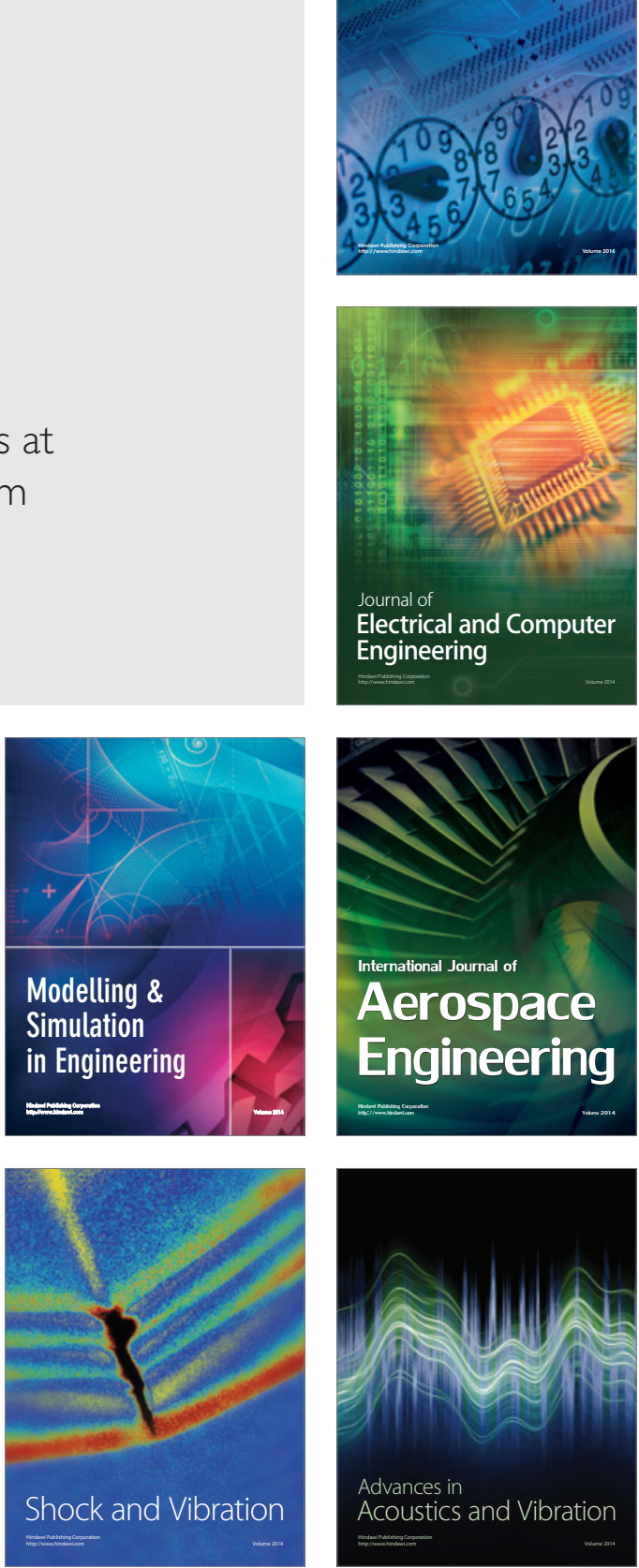